\title{
A MORPHOLOGICAL SUBBAND DECOMPOSITION STRUCTURE USING GF(N) ARITHMETIC
}

\author{
Metin Nafi Gürcan, Ömer N. Gerek, A. Enis Cetin \\ Bilkent University, Dept. of Electrical and Electronics Engineering, \\ Bilkent, Ankara TR-06533, Turkey \\ E-mail: gurcan@ee.bilkent.edu.tr
}

Phone: (90) 312-266 4307 Fax: (90) 312-266 4126

\begin{abstract}
Linear filter banks with critical subsampling and perfect reconstruction ( $\mathrm{PR}$ ) property have received much interest and found numerous applications in signal and image processing. Recently, nonlinear filter bank structures with PR and critical subsampling have been proposed and used in image coding. In this paper, it is shown that PR nonlinear subband decomposition can be performed using the Gallois Field (GF) arithmetic. The result of the decomposition of an n-ary (e.g. 256-ary) input signal is still $n$-ary at different resolutions. This decomposition structure can be utilized for binary and $2^{k}$ ( $k$ is an integer) level signal decompositions. Simulation studies are presented.
\end{abstract}

\section{INTRODUCTION}

The subband decomposition is widely used in coding and signal analysis. Traditionally, linear filters with PR property and critical subsampling are employed for the decomposition. However nonlinear filters were not used for such purposes, mainly due to the lack of frequency domain tools readily available for linear systems. Recently, some nonlinear subband decomposition structures with $\mathrm{PR}$ and critical subsampling were proposed and used in image coding [1] - [4]. In these decompositions, the key idea is to replace the linear filters in a classical subband decomposition scheme with nonlinear filters.

This work is supported by TUBITAK (Turkish Scientific and Technical Research Council) Grant No. COST 249, and NSF Grant No. INT-9406954.
In this paper it is shown that $P R$ nonlinear subband decomposition can be performed using the Gallois Field (GF) arithmetic. The result of the decomposition of an n-ary (e.g. 256-ary) input signal is still $n$-ary at different resolutions. Without using the GF arithmetic, after one level of subband decomposition an image with with 256 gray levels ( 0 to 255 ) produces subband images which have pixel values in the range $[-255,255]$. The range increases as the number of levels increases.

The GF(N) arithmetic is also used in the wavelet theory [5] - [8]. In [5] a framework for the wavelet transform of Finite Cyclic Groups was developed. In [6] this idea was specifically used for the binary decomposition of binary images. The generalization of wavelet decomposition and perfect reconstruction filter banks to finite fields were also discussed in $[7,8]$.

In Section 2 nonlinear subband decomposition in $\mathrm{GF}(\mathrm{N})$ arithmetic is introduced. In Section 3 simulation examples are presented, and in Section 4 image analysis using bit planes is discussed.

\section{NONLINEAR DECOMPOSITION IN GF(N) ARITMETIC}

The nonlinear subband decomposition of signal $x$ is carried out using the structure shown in Figure 1. In this structure $\mathcal{H}$ and $\mathcal{G}$ are nonlinear operators, and they produce $y_{a}$ (analysis) and $y_{d}$ (detail) signals from the input signals $x_{1}(n)=x(2 n-1)$ and $x_{2}(n)=x(2 n)[4]$ :

$$
y_{d}(n)=x_{2}(n)+\mathcal{H}\left(\mathbf{x}_{1}(n)\right)
$$




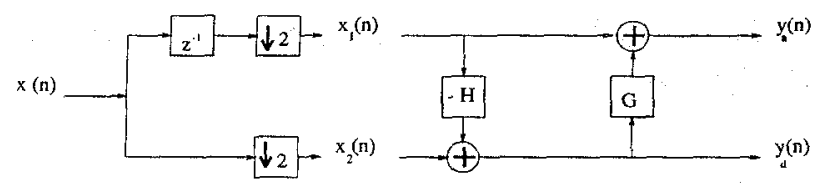

Figure 1: n-ary Subband Decomposition Structure

$$
y_{a}(n)=x_{1}(n)-\mathcal{G}\left(\mathbf{y}_{d}(n)\right)
$$

where

$\mathbf{x}_{1}(n)=\left(x_{1}\left(n-N_{1}\right), \ldots, x_{1}(n), \ldots, x_{1}\left(n+N_{2}\right)\right)$, $\mathbf{y}_{d}(n)=\left(y_{d}\left(n-N_{2}\right), \ldots, y_{d}(n), \ldots, y_{d}\left(n+N_{3}\right)\right)$,

and $N_{1}, N_{2}, N_{3}, N_{4}$ are positive integers.

The PR property of this nonlinear decomposition structure is preserved if the GF arithmetic is used. The results of the nonlinear operations have no effect on the PR property as long as they produce outputs in $G F(N)$ because these are cancelled out in the synthesis part.

For the above analysis equations, the corresponding synthesis equations are:

$$
\begin{aligned}
& x_{1}^{\prime}(n)=y_{a}(n)-\mathcal{G}\left(\mathbf{y}_{d}(n)\right) \\
& x_{2}^{\prime}(n)=y_{d}(n)+\mathcal{H}\left(\mathbf{x}_{1}(n)\right)
\end{aligned}
$$

The following set of equations straightforwardly shows that PR property is preserved in the GF arithmetic:

$$
\begin{aligned}
x_{1}^{\prime}(n) & =y_{a}(n) \Theta \mathcal{G}\left(\mathbf{y}_{d}(n)\right) \\
& =x_{1}(n) \oplus \mathcal{G}\left(\mathbf{y}_{d}(n)\right) \Theta \mathcal{G}\left(\mathbf{y}_{d}(n)\right) \\
& =x_{1}(n)
\end{aligned}
$$

where $\theta, \oplus$ represent the GF subtraction and addition operations, respectively. Since $x_{1}^{\prime}(n)$ is equal to $x_{1}(n)$ the nonlinear decomposition structure provides $P R$ in the $G F(N)$ arithmetic, as well.

\section{BINARY AND N-ARY SUBBAND DECOMPOSITIONS}

The binary subband decomposition of binary images such as bit-plane images or textual images

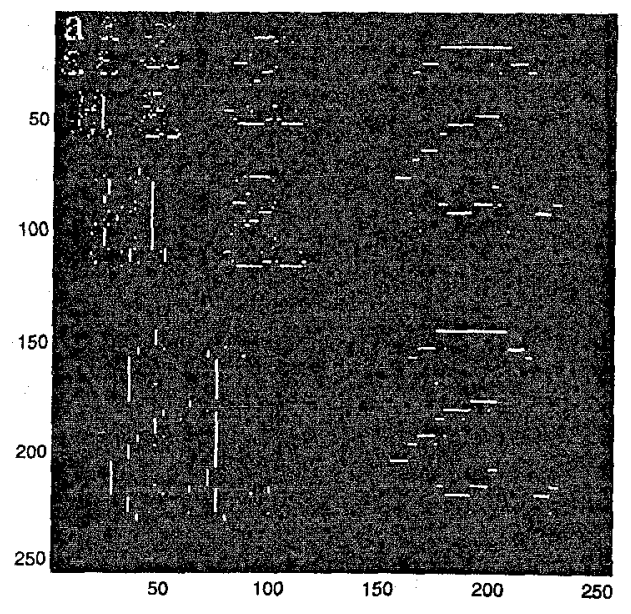

Figure 2: Binary Subband Decomposition of the letter ' $a$ ': Morphological Subband Decomposition



Figure 3: Binary Subband Decomposition of the letter 'a': The Binary Wavelet Transform

can be accomplished by utilizing the nonlinear subband decomposition structure with the GF(2) arithmetic. Figure 2 illustrates the binary decomposition of the letter "a"; Figure 3 shows the binary wavelet decomposition (BWT) of the same image. The ringing effects present in the BWT disappear in the nonlinear subband decomposition.

It is possible to develop lossless binary coding methods based on binary decomposition structure. In one such method, subband images are encoded using embedded zero-tree coding and entropy coding [9].

Figure 4 shows the subband decomposition of $512 \times 512$ Lena image which has 256 gray levels. 
The decomposition is obtained using the nonlinear structure depicted in Figure 1 and by taking $\mathcal{H}\left(\mathbf{x}_{1}(n)\right)=\operatorname{median}\left(\mathbf{x}_{1}(n)\right)$ and $\mathcal{G}\left(\mathbf{y}_{d}(n)\right)=0$. This case corresponds to the nonlinear decomposition structure developed by Egger and Kunt [1] performed in the $\operatorname{GF}(\mathrm{N}=256)$ arithmetic. The low-low subband image is obtained by directly downsampling the image. Ringing effects present in linear-filter decompositions do not appear in the low-high, high-low, and high-high subimages. This may especially be useful for pattern matching applications in subbands.

Another application is lossless image compression. For instance, the most signilicant bit plane of the $256 \times 256$ Lena image could be losslessly compressed 3.01 times after nonlinear subband decomposition whereas the Lempel-Ziv coding resulted in a compression ratio of 2.44 [9]. This finite field decomposition structure can also be extended to coding $k$ (e.g. 256) level images provided that the $\mathrm{GF}(\mathrm{k})$ arithmetic can be performed.

\section{BIT PLANE SUBBAND DECOMPOSITION}

A $2^{k}$ level signal decomposition can be parallely implemented in bit planes, which can be utilized in some VLSI implementations. In this section conditions for the parallel implementation are described.

Nonlinear subband decomposition in bit planes is achieved by first dividing the $2^{k}$ level signal into $k$ bit planes and then decomposing each bit plane separately in parallel. Finally, the outputs of the decompositions are combined together to obtain the $2^{k}$ level decomposition.

The sub-signal obtained by combining the bit planes is not always equivalent to the sub-signal obtained by direct subband decomposition. The necessary conditions for the equivalence can be developed as follows. Consider the nonlinear subband decomposition procedure of Section 2. If we repeat the procedure for each bit plane then we get:

$$
y_{d i}(n)=x_{2 i}(n) \Theta \mathcal{H}\left(\mathbf{x}_{1 i}(n)\right)
$$

$$
y_{a i}(n)=x_{1 i}(n) \oplus \mathcal{G}\left(\mathbf{y}_{d i}(n)\right)
$$

where $x_{1 i}$ represents the $i$-th bit plane of the signal $x_{1}$, etc.

The bit planes are added together to get the subband decomposed signal.

$$
\begin{aligned}
y_{d}(n) & =\sum_{i=1}^{N} y_{d i}(n) \times 2^{i} \\
& =\sum_{i=1}^{N} x_{2 i}(n) \times 2^{i} \\
& \ominus \sum_{i=1}^{N} \mathcal{H}\left(\mathbf{x}_{1 i}(n)\right) \times 2^{i} \\
& =x_{2}(n) \ominus \sum_{i=1}^{N} \mathcal{H}\left(\mathbf{x}_{1 i}(n)\right) \times 2^{i}
\end{aligned}
$$

where $N$ is the number of bit planes. If

$$
\sum_{i=1}^{N} \mathcal{H}\left(\mathbf{x}_{1 i}(n) \times 2^{i}\right)=\mathcal{H}\left(\sum_{i=1}^{N} \mathbf{x}_{1 i}(n) \times 2^{i}\right)(13)
$$

and

$$
\sum_{i=1}^{N} \mathcal{G}\left(\mathbf{x}_{1 i}(n) \times 2^{i}\right)=\mathcal{G}\left(\sum_{i=1}^{N} \mathbf{x}_{1 i}(n) \times 2^{i}\right)(14)
$$

then the direct subband decomposition and subband decomposition in bit planes will yield the same decomposed signals. This condition is satisfied for some filters such as median filters. One additional advantage of using median type filters is that the output of such filters can be efficiently computed if the input signal is binary. This can be accomplished by first summing the signal values of the input to the median filter and then comparing the sum with a threshold which is set at half the input signal length. If the sum is greater than the threshold the output is one, otherwise it is zero.

The decomposition structure can be extended to two-dimensions for the rectangular and quincunx sampling cases. For this purpose separable filters can be used and the input signal is filtered horizontally and vertically by these filters. Another possibility is to use two dimensional filters. Figure 5 shows an example region of support for such filtering. 


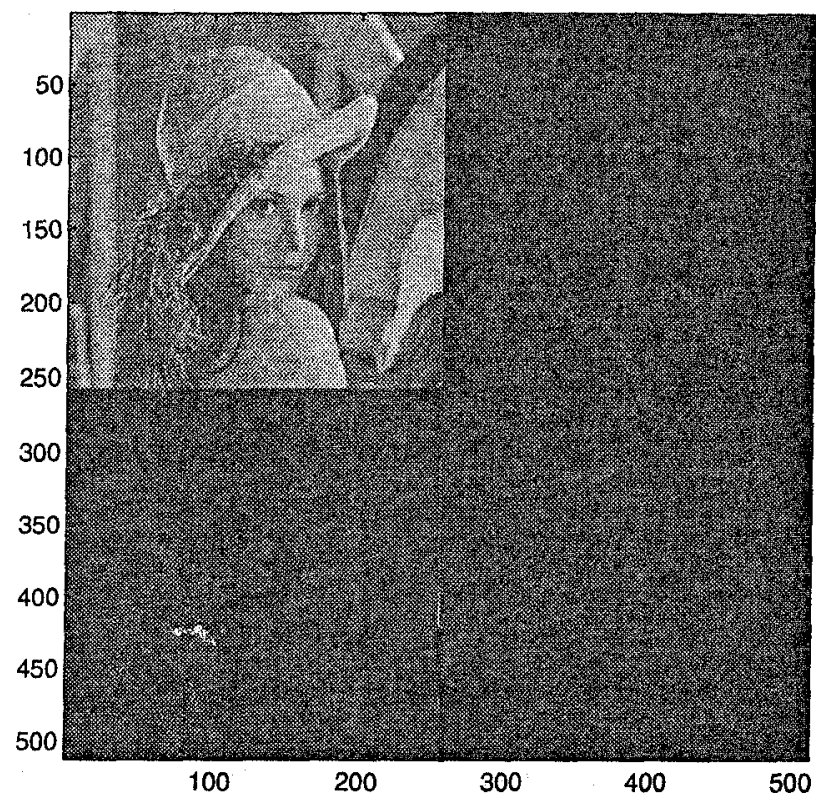

Figure 4: 256-ary Subband Decomposition of Lena Image

\section{CONCLUSION}

In this paper we present a nonlinear subband decomposition structure employing the $\mathrm{GF}(\mathrm{N})$ arithmetic. The subband images in this structure preserve the gray-scale level of the original image. The decomposition structure results in binary (256 gray level) subband images for binary (256 gray level) images. It is possible to parallelize the decomposition structure in the $G F(N)$ arithmetic for certain types of nonlinear filters in bit planes. This results in an efficient realization of the nonlinear subband decomposition.

\section{REFERENCES}

[1] O. Egger, M. Kunt, "Embedded Zerotree Based Lossless Image Coding," IEEE ICIP'95, vol. II pp. 616-619., June 1995.

[2] D. E. F. Florencio, R. Schafer "Perfect Reconstructing Nonlinear Filter Banks," in ICASSP'96, 1996.

[3] R. L. de Queiroz, D. A. F. Florencio, "A Pyramidal Coding Using a Nonlinear Filter Bank," in ICASSP'96, 1996.

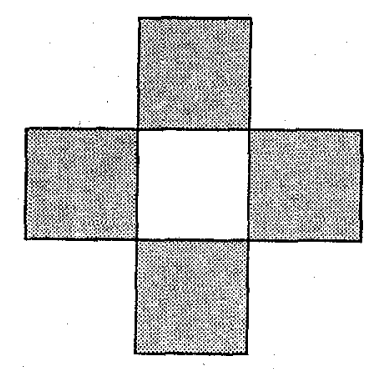

\section{REGION OF SUPPORT}

\section{CURRENT PIXEL}

Figure 5: Region of Support for two dimensional nonlinear filtering

[4] F. J. Hampson, J. C. Pesquet, "A Nonlinear Subband Decomposition with Perfect Reconstruction," in ICASSP'96, 1996.

[5] G. Caire, R. L. Grossman, H. V. Poor, "Wavelet Transforms Associated with Finite Cyclic Groups," IEEE Transactions on Information Theory, vol. 39, No. 4, July 1993.

[6] M. D. Swanson and A. H. Tewfik, "A Binary Wavelet Decomposition of Binary Images," Submitted to IEEE Trans. Image Processing (IP-941).

[7] P.P. Vaidyanathan, "Unitary and Paraunitary Systems in Finite Fields," in Proc. 1990 IEEE Int. Symp. on Circuits and Systems, New Orleans, LA, pp. 1189-1192, 1990.

[8] P.P. Vaidyanathan, Multirate Systems and Filter Banks, Englewood Cliffs, N.J, Prentice Hall, 1992.

[9] M. N. Gürcan, Ö. N. Gerek, A. E. Çetin, "Binary Morphological Subband Decomposition For Image Coding," TFTS-Symposium, 1996. 